\title{
Tendencias religiosas en la antroponimia rondonense
}

\section{Religious trends in the anthroponomy of Marechal Cândido Rondon city}

Patrícia Helena Frai

Universidade Estadual do Oeste do Paraná

Doutoranda do Programa de Pós-Graduação em Letras

http://orcid.org/0000-0003-0961-0051

patríciafrai@hotmail.com

Traduzido por

Yolanda Guillermina López Franco

\section{Resumen}

https://orcid.org/0000-0002-9306-6564

Es de sabiduría popular que atribuir nombres religiosos a los hijos puede significar devoción a un Santo, personaje bíblico o creencia religiosa particular de una determinada comunidad. Sin embargo, al saber de hecho las motivaciones reales para elegir un determinado antropónimo, a partir de entrevistas con los padres o con los propios portadores del nombre propio, se observa que los nombres religiosos pueden evidenciar cuestiones más allá de la simple devoción. En ese sentido, el objetivo de este artículo es analizar, a partir de los estudios de la socioantroponomástica, qué factores influencian en la práctica de nombrar a un hijo con el modelo de atribución denominado religión (JIMÉNEZ SEGURA, 2014). Para ello, la investigación emplea un corpus formado por 250 nombres yuxtapuestos colectados a partir de entrevistas semiestructuradas, en un lapso que abarca 1930-1940 a 2010, en la ciudad de Marechal Cândido Rondon, Brasil. La investigación apunta que atribuir nombres religiosos puede formar parte de un ideario de una determinada comunidad, ya sea como nominación de un hijo, a través de un nombre bíblico, puede mostrar cuestiones identitarias más allá del significado restringido del nombre (SEIDE, 2016).

Palabras clave: Socioantroponomástica; motivación religiosa; nombres yuxtapuestos.

\begin{abstract}
It is popular wisdom that giving religious names to children can mean devotion to a saint, a biblical character, or a particular religious belief in a particular community. However, when knowing in fact the real motivations for a specific anthroponym, from interviews with parents or the name bearers, it is observed that religious names can highlight issues beyond simple devotion. In this direction, the aim of this article is to analyse, from the studies of Socioanthroponomics, which factors influence the naming of a child with the traditional attribution model called as religion (JIMÉNEZ SEGURA, 2014). To this end, the research uses a corpus of 250 juxtaposed names collected from semi-structured interviews, in a span of time ranging from 1930-1940 to 2010, in the city of Marechal Cândido Rondon, Brazil. The research points out that assigning religious names can be part of an ideal of a certain community, as well as the naming of a child, using a biblical name, can highlight identity issues beyond the meaning of the name itself (SEIDE, 2016).
\end{abstract}

Key Words: Socioanthroponymics; Religious motivation; Juxtaposed names. 


\section{Introducción}

Los nombres propios de persona revelan marcas identitarias y culturales de una comunidad. Es bajo este enfoque que la socionomástica estudia los nombres propios de persona, también denominados aquí antropónimos. Estudiar tales nombres ha sido el objetivo de diversas investigaciones del área de Letras, Sociología, Historia, Antropología, entre otras. La gran diferencia es el enfoque que estas disciplinas pueden dar a tales estudios, esto es, los antropónimos como medio para llegar un resultado o emplear los nombres propios como un fin, teniendo en cuenta el carácter de los estudios en el área de Letras (SEIDE, 2016) ${ }^{1}$.

En esta dirección se encuentra la perspectiva teórica del presente artículo. Se cree que los nombres propios de persona, además de signos lingüísticos, pueden ser una gran fuente de estudios de la sociedad. Ello demuestra que los antropónimos no ejercen solamente la función meramente distintiva y designativa, como se señalaba en las primeras investigaciones en el área de la antroponomástica (Mill, apud DICK, 1992: 181). Para el autor, los nombres tendrían apenas la función de diferenciar a un individuo de otro y no implicaban ningún significado. Esta idea del nombre propio restringe los estudios del área pues acaban volviéndose sólo una marca de identificación personal (DICK, 1992: 181), lo que desconsidera otros sentidos que puedan connotar.

Dick (1992) amplía esa percepción, al considerar los estudios de la antroponomástica en Brasil y afirmar que tales piezas léxicas son, realmente significativas y connotativas (DICK, 1992: 181). La elección del nombre propio de persona para se lo atribuir a un hijo pone en evidencia creencias que, en un determinado momento, los padres tenían. En esta

\footnotetext{
${ }^{1}$ Otros enfoques de investigaciones antroponomásticas pueden realizarse en el ámbito social como la Antroponomástica Comparada (SEIDE, 2020a). La perspectiva de la comparación antroponímica ocurre frente a la necesidad de comprender los movimientos migratorios y sus consecuencias en la lenguas de uso, lo que provoca muchas veces la traducción de los antropónimos.
} 
perspectiva de estudio surge la socionomástica, que tiene como objeto el estudio de los nombres propios bajo la óptica social, cultural e identitaria (VAN LANGENDONCK, 2007). Así, se observa que hay diferentes motivaciones que llevan a los padres a atribuir un determinado nombre para su hijo, las cuales pueden ser religiosa, de homenaje a algún familiar o amigo, por influencia mediática, por circunstancias de nacimiento, entre otras (GUÉRIOS, 1981). Además, existen trabajos que se enfocan en entender los cambios de estas elecciones en una determinada comunidad, como Pensin (2020), que logró mostrar cómo el perfil denominador de los habitantes en diferentes momentos puede ser distintos e influir en los cambios entendidos sobre un eje cronológico.

Considerando esa perspectiva de estudio, el presente artículo tiene como objetivo verificar la frecuencia de uso del modelo de atribución tradicional religioso en nombres yuxtapuestos femeninos y masculinos en la ciudad de Marechal Cândido Rondon, Estado de Paraná, Brasil. Se empleó inicialmente un corpus de 250 nombres yuxtapuestos, generados a partir de entrevistas semiestructuradas de una investigación de maestría defendida en 2016. Se dividieron tales nombres por partes iguales entre los géneros femenino y masculino, en un corte diacrónico que contempla las décadas de 1930/1940, 1950, 1960, 1970, 1980, 1990, 2000 y 2010. Cabe señalar que se consideran como antropónimos yuxtapuestos aquellos constituidos por dos piezas léxicas, atribuidos al hijo de manera, muchas veces, no usual, por ejemplo, Maria Bruna y Pedro Henrique. El primer nombre será designado aquí como n1 y el segundo, como n2.

También es objetivo de esta investigación el observar a la luz de la socionomástica algunas hipótesis explicativas sobre el porqué del uso de tales nombres, observando la incidencia de la motivación en cada género, y observar las razones por las que tales elecciones pudieran haberse producido en el período temporal de análisis. En este sentido, se plantean algunas preguntas orientadoras, coincidentes con una investigación de Seide (2016), con el 
fin de que sea posible analizar el corpus: ¿La frecuencia mayor de nombres religiosos en una determinada década puede comprobar una mayor religiosidad? En sentido inverso ¿ si hay una década con menor mención de la motivación religiosa, sería pertinente afirmar que las personas del municipio se volvieron menos religiosas?

Para el trabajo que ahora se presenta, se seleccionó solamente el número de ocurrencias del modelo de atribución tradicional "religión", tanto las ocurrencias en n1 y n2 femenino, como n1 y n2 masculino. Se categorizó así la motivación a partir de las informaciones aportadas por los entrevistados, es decir, para la categorización de los antropónimos, la investigación se valió de las narrativas hechas por los informantes, sobre aquello que saben u oyeron de sus padres.

Así, el artículo delineará primeramente el soporte teórico que emplea la investigación; en seguida, se presenta la metodología adoptada para el análisis. A continuación, se realiza el análisis cuantitativo de los datos y, por consiguiente, las hipótesis que pueden explicar los números obtenidos. El último elemento del artículo son las consideraciones finales.

\section{La motivación religiosa en la socionomástica}

Los nombres propios pueden ser estudiados bajo dos perspectivas diferentes: la primera, bajo el enfoque estrictamente lingüístico y, la segunda, desde el aspecto social y cultural (GUÉRIOS, 1981: 16). Las investigaciones que adoptan el primer enfoque se basan normalmente en análisis documentales; su objetivo es estudiar, por ejemplo, la etimología y el origen del nombre. En tanto que las investigaciones que adoptan la segunda perspectiva necesitan hacer entrevistas y saber el porqué de la elección de los nombres, es decir, por qué 
los padres atribuyen determinado nombre al hijo y cuáles son los factores que los hicieron escoger determinado nombre y no otro.

Para entender los factores sociales y culturales que influyen en la norma antroponímica de un determinado lugar, se observa que las investigaciones del área de la socionomástica emplean la categorización antroponímica, que culmina en las motivaciones de los nombres propios de persona.

De acuerdo con Seide (2018), la categoría antroponímica se divide en antropónimos cuya motivación es tradicional o perteneciente a la moda. La autora define que los nombres tradicionales son aquellos que se refieren a los valores morales, que pasan de generación en generación, como también pueden ser aquellos que se emplean habitualmente y son considerados tradicionales en un determinado lugar (SEIDE, 2018: 168). Tal categoría se subdivide en las seguientes motivaciones: nombres religiosos y nombres que son homenaje a los familiares. La segunda categoría son los antropónimos muy utilizados en un determinado momento, forman parte del gusto colectivo, pero que súbitamente pueden dejarse de emplear, con lo que tienen intervalos temporales de uso. Las motivaciones consideradas en la moda son la influencia mediática -nombres de personajes famosos y nombres atribuidos debido a la estética: sonido, rima, ortografía).

En lo que se refiere a los nombres tradicionales, la motivación religiosa es el objeto de estudio de este artículo. Se consideran como nombres cuya motivación es religiosa, aquellos que fueron previamente mencionados a) devoción a un santo: los padres atribuyen el nombre al hijo porque la familia es devota de algún santo católico; b) nombre bíblico: atribución de nombres de personajes de la biblia; c) nombre atribuido por el calendario litúrgico: cuando el niño nace el día de un santo y, por eso, se lo nombra con tal antropónimo; d) nombre de padrinos o atribuido por los padrinos: se considera que los padrinos son 
escogidos para el bautismo del niño y, por eso, están asociados con la educación cristiana del individuo.

\section{La motivación religiosa en antropónimos femeninos y masculinos}

Para verificar la cantidad de ocurrencias del modelo tradicional religioso de los nombre propios, se contabilizó el número de casos en n1 y n2 femenino, así como en n1 y n2 masculino. Cada gráfico presenta separadamente el número en porcentaje de motivación religiosa para ambos géneros, considerando el total de ocurrencias. Primeramente, se hizo una comparación entre n1 y n2 femenino y, después, entre n1 y n2 masculino. En seguida se cruzarón los datos, con el fin de que se puediese visualizar las posibles diferencias en el comportamiento de la motivación en nombres femeninos y masculinos. Cabe informar que los datos fueron contabilizados en porcentajes con el fin de que se puedieran volver comparables los datos medidos.

Por lo que se refiere a los nombres yuxtapuestos femeninos, se encontró un total de 21 menciones para n1 femenino y 36 para n2. El siguiente gráfico ilustra la frecuencia porcentual de la motivación en cada década.

\section{Gráfico 1}

La motivación religiosa en $n 1$ y n2 femenino 


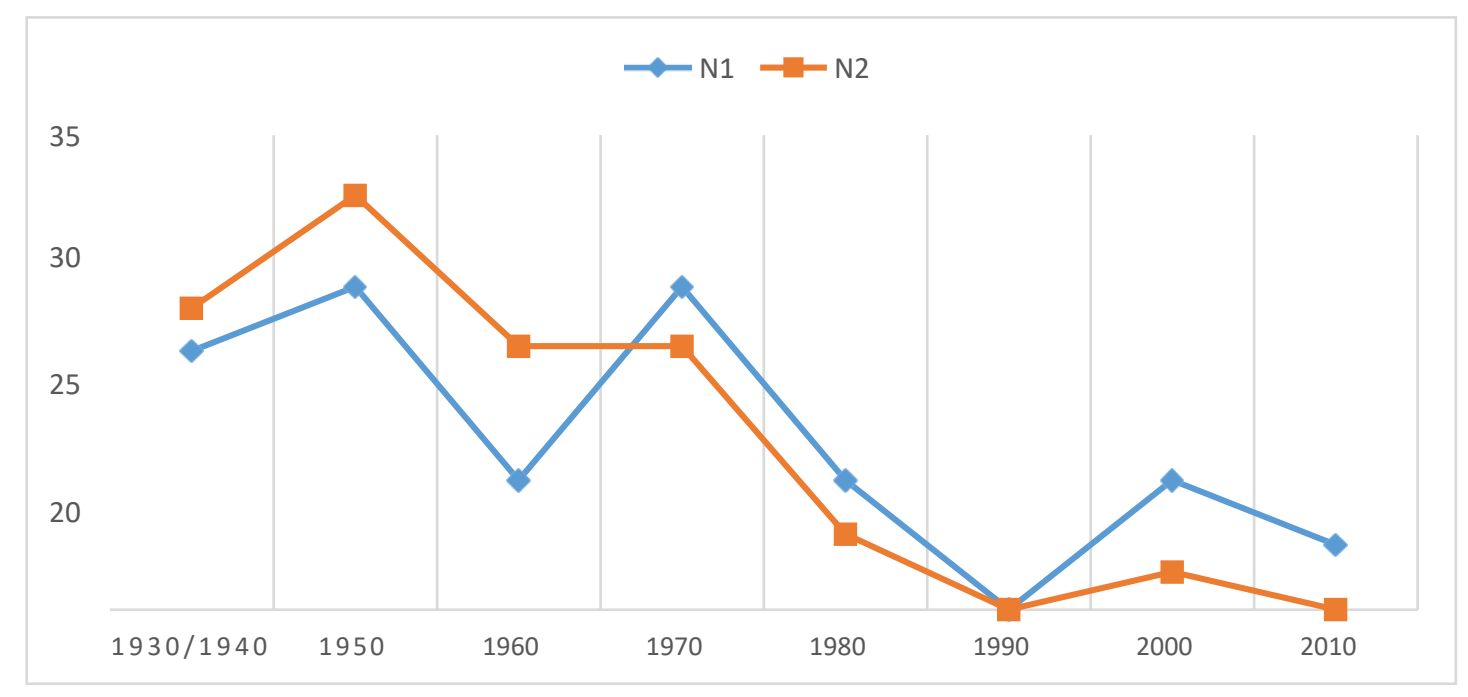

Fuente: elaborado por la autora (2019)

A partir del recorte diacrónico es posible observar que la motivación religiosa posee ocurrencias distintas en las décadas de análisis. En 1930/1940, es posible observar que el n1 femenino obtiene un porcentaje de 19\%; alcanza un pequeño crecimiento de $4 \%$ en la década posterior, con lo que suma 23\%. En la década de 1960, la motivación religiosa decae al 9\%, no obstante, en 1970 vuelve a ser mencionada y llega al máximo de ocurrencias, con lo que iguala a la década de 1950: 23\% de los casos. Después de 1970, el porcentaje cae en las décadas siguientes: en 1980 es de $9 \%$ y en 1990 no hay ningún caso con esta motivación. En 2000, la motivación vuelve a ser mencionada en 9\% de las ocurrencias y, en 2010, solo en $4 \%$ de los casos.

En el n2, el comportamiento en el inicio del recorte temporal es semejante al del n1. En 1930/1940, el n2 obtuvo un porcentaje de 22\%, 3\% más, si se compara con n1. En la década de 1950, se obtuvo el mayor crecimiento, con lo que la motivación alcanza el 33\% del total de ocurrencias, siendo así 10\% mayor que n1. Aunque el número de casos sea diferente entre $\mathrm{n} 1$ y n2, se observa que el comportamiento de incrementos y decrementos es semejante. En la siguiente década, la religión tiene el 19\% de los casos, si bien represente un decrecimiento en relación con la década anterior, todavía el número de ocurrencias es mayor 
al alcanzado por el n1 (9\%). Ese porcentaje se mantiene constante en la década de 1970, en que la proporción observada es del 19\%. En este punto, en tanto que en n2 se mantiene constante la motivación religiosa, en n1, el número de casos aumenta en 14\%; los datos apuntan hacia una dinámica mayor en n1 que en n2.

En 1980, la motivación religiosa en el n2 sufre una caída significativa de $23 \%$ en relación con la década anterior, que es apenas del 9\% y, en 1990, no hay ninguna mención de la motivación religiosa. Este mismo hecho puede observarse en n1. De nuevo se observan semejanzas en el comportamiento de las ocurrencias. En las últimas décadas, en 2000 hay un aumento irrisorio del 2\% y regresa a la posición cero en 2010.

Frente a los datos descritos, es notorio que hay periodos en que la motivación religiosa pose un comportamiento semejante tanto para el n1 como para el n2. En otras palabras, en las décadas en que hay aumento de tal motivación en el n1, lo mismo ocurre en el n2; en momentos en que en el n1 disminuyen las ocurrencias, en n2 también disminuyen. Sólo en un período el comportamiento diverge: entre 1960 y 1970. En tanto que para el n1 hay aumento de la motivación, en el n2, la mención de la motivación se mantiene constante.

El gráfico siguiente señala los números referentes a n1 y n2 masculinos.

\section{Gráfico 2}

Motivación religiosa en n1 y n2 masculino 


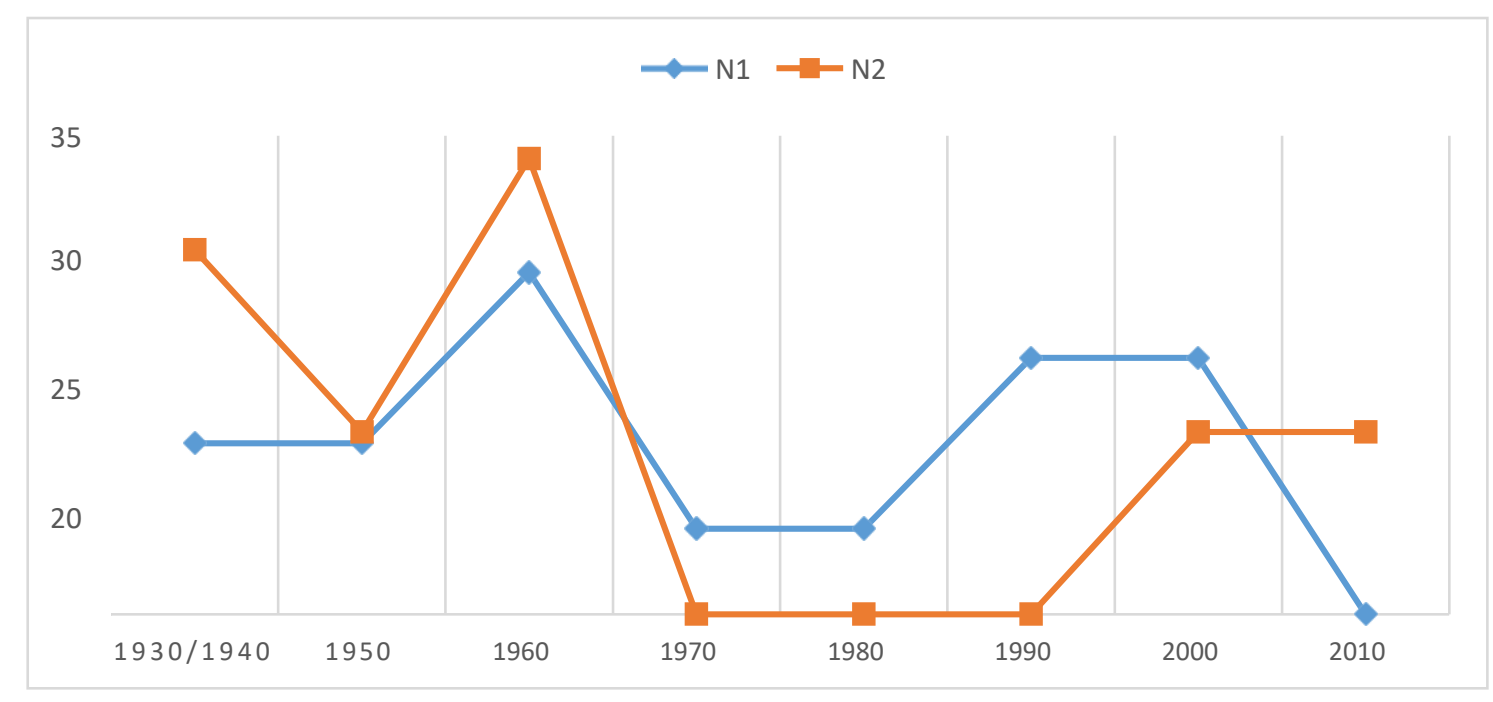

Fuente: elaborado por la autora (2019)

En la década de 1930/1940, n1 inicia con 12\% de las ocurrencias; el mismo porcentaje se extiende a 1950, con 13\%. En la década siguiente, en 1960, hubo un aumento del 12\%: el mayor porcentaje de la motivación religiosa para n1 masculino se produce en esta década, totalizando $25 \%$ de las ocurrencias. Por consiguiente, hay una caída al $19 \%$ en el porcentaje entre la década en cuestión y 1970. Así se continúa hasta 1980, cuyo porcentaje también es de 6\%. De 1980 a 1990 hay un aumento en el número de ocurrencias, que alcanzan 18\%, y se mantiene constante hasta 2000. Por fin, hay una caída brusca en 2010, en el que la motivación religiosa no tiene ningún caso.

Para n2, la motivación religiosa obtuvo 26\% de las ocurrencias en 1930/1940 y, en la década posterior sufrió una caída hasta el 13\%, prácticamente el mismo porcentajes que inició las dos primeras décadas para n1. En la del 1960 se presenta la mayor proporción de la motivación religiosa para $\mathrm{n} 2$ masculino: $33 \%$ - una diferencia del $8 \%$ con respecto a $\mathrm{n} 1$. Se observa que en 1960 se ubica el mayor número de casos de motivación religiosa, tanto para n1 como para n2. Si en esa década se presenta el mayor número, en la década posterior, 1970, no hubo ninguna mención de los nombres religiosos y ese hecho se extiende por tres décadas consecutivas: 1970, 1980 y 1990. A diferencia de lo que ocurre en n1: estancamiento entre 1970 y $1980(6 \%)$ y aumento de 1980 a 1990 del 12\%. En la década de 2000, regresan los 
nombres de motivación religiosa: su proporción se eleva al 13\% y se mantiene constante en 2010.

Como se puede ver, a partir del gráfico, que el comportamiento del modelo de atribución tradicional religioso, incluso con porcentajes diferentes, ora converge, ora diverge. El intervalo entre 1950-1980 posee semejanzas: en la década en que los porcentajes aumentan para el n1, lo mismo ocurre para el n2. A partir de 1980 los datos divergen: mientras que para n2 la motivación religiosa no aparece de 1980 a 1990, en n1 el porcentaje aumenta al 18\%. Hay otro momento similar en las últimas décadas: para el n1, de 2000 a 2010, la motivación decae al $18 \%$ y llega a cero menciones, mientras que para n2, se mantiene constante en $13 \%$.

\section{Tendencias en la motivación religiosa en antropónimos femeninos y masculinos}

Se deben hacer algunas consideraciones sobre los principales datos ilustrados en los gráficos, con el fin de que puedan ser objeto de reflexión en esta sección. El primer punto que se destaca es el modelo de atribución nominal tradicional "religión", predominante en el n1 femenino. El porcentaje es más elevado en las décadas 1930/1940, 1950, 1970, 1980 y 2010. Por lo que se refiere al n2, en los nombres masculinos, la motivación no fue mencionada en tres décadas consecutivas, mientras que para n2 femenino, son dos décadas consecutivas (1990 y 2010). Tampoco se mencionó la motivación religiosa en el n1 masculino en la década 2010, mientras que, en esa misma década, se alcanzó el 13\% en esa motivación en el n2, con lo que se muestra que muchas veces la disminución de la motivación puede ser, ora en el n1, ora en el n2. Es decir que no siempre se atribuyeron con la misma motivación los n1 y n2. En los nombres femeninos, el intervalo en el que no hubo casos de motivación religiosa tanto en n1 como en n2, es sólo en 1990, hecho éste que diferencia el comportamiento atributivo observado en los nombres masculinos. 
A pesar de que se hayan hecho algunas observaciones, queda de manifiesto que la motivación religiosa, tanto en los nombres femeninos como en los masculinos, decreció visiblemente para ambos sexos en $1980 \mathrm{y}$, principalmente, en 1990, aunque tuvo un incremento pequeño en las décadas posteriores. Los números revelan que, por ejemplo, no hubo ninguna ocurrencia de nombres religiosos en los femeninos, tanto en $\mathrm{n} 1$, como en $\mathrm{n} 2$; $\mathrm{y}$ para los nombres masculinos, hubo sólo tres casos, lo que puede ser considerado como un valor menor en comparación con otras décadas. Con base en estas afirmaciones, surgen algunos cuestionamientos acerca de tales comportamientos: ¿habría una pérdida de la religiosidad en la ciudad de Marechal Cândido Rondon, que se pone de manifiesto en la disminución de la motivación religiosa? ¿El retorno de esas motivaciones sería un retorno de los valores religiosos? ¿Por qué nombres de motivación religiosa fueron atribuidos a principalmente a mujeres en las décadas 1930/1940 y $1950 ?$

En un artículo sobre la identidad religiosa Seide (2016) compara el uso de los nombres que son pretendidamente religiosos, esto es, que poseen significado religioso, con aquellos que poseen una motivación religiosa, en la ciudad de Marechal Cândido Rondon. Para ello, la autora emplea tres corpora diferentes de análisis: actas de nacimiento resguardadas en la oficina del Registro Civil de la ciudad en el período de 1961 a 2001, narrativas y cuestionarios respondidos por alumnos de la licenciatura de Letras, del año de 2012 a 2013. La autora se sirve del método cualitativo para el análisis de los nombres, bajo una perspectiva interdisciplinaria para entender el fenómeno del uso de los nombres supuestamente religiosos. Inicialmente fueron separados los nombres de los académicos cuyo lema era religioso, como, por ejemplo, José, Cristina, Teresinha y Fátima. Se averiguó su significado en diccionarios etimológicos, como el de Guérios. En seguida, tales antropónimos fueron comparados con los nombres encontrados en las actas de nacimiento. La comparación mostró convergencia de uso: los mismos nombres atribuidos a los académicos se encuentran en las actas de nacimiento 
en el respectivo año de atribución. El nombre Cristina, encontrado en las narrativas de los académicos fue dado a personas nacidas en 1980 y 1990, mismas décadas en que Cristina se documenta en los registros de Marechal Cândido Rondon (SEIDE, 2016: 340).

Con base en la gran cantidad de nombres pretendidamente religiosos encontrados en los relatos, cuestionarios y actas de nacimiento es posible notar una tendencia al empleo de tales antropónimos. Sin embargo, otro factor encontrado es si tales nombres fueron atribuidos realmente por ser nombres religiosos. En ese sentido, se observó que gran parte de los nombres pretendidamente religiosos fueron atribuidos por motivaciones distintas, hecho comprobado a partir de las narrativas de los informantes. El nombre Crystine, derivado de Cristina, fue otorgado, según la entrevistada, porque a los padres les pareció bonita y llamativa la sonoridad del nombre. (SEIDE, 2016: 341). De los 15 nombres analizados, solamente dos fueron atribuidos realmente por su valor semántico, hecho datado para las décadas de 1980 y 1990, las mismas que no presentan motivación religiosa en la presente investigación.

De acuerdo con Seide (2016), atribuir nombres religiosos a los hijos a partir de su lema es una forma de subrayar la identidad religiosa de la familia hacia el hijo. El empleo de la motivación religiosa al atribuir un nombre indica una identidad que los padres desean pasar al hijo. De esa manera, para entender tal fenómeno, Seide (2016) apunta algunas hipótesis que pueden justificar los resultados. Para ello fue necesario entender si en la ciudad de Merechal Cândido Rondon existe un decremento en la identificación de la religiosidad y si la expresión de la identidad ya no se revela a partir de los antropónimos, sino de otras maneras.

Las mismas hipótesis pueden formularse con base en los resultados aquí analizados. ¿Sería posible que el hecho de no haber menciones de los nombres religiosos en la década 1990, tanto para n1 como para n2 femeninos, y no haber casos en tres décadas consecutivas 
para 2 masculinos pudiera estar relacionado con la pérdida de la religiosidad? Es importante subrayar en este punto que no son objeto de estudio de este artículo los nombres pretendidamente religiosos, aunque se puedan realizar posteriormente investigaciones bajo esa perspectiva. El foco está aquí en entender si tal hipótesis también puede aplicarse al fenómeno que se destaca en este trabajo, dado que se trata de la misma ciudad estudiada, así como el mismo corte temporal.

Para poner a prueba la primera hipótesis, Seide (2016) emplea el censo del IBGE [Instituto Brasileño de Geografía y Estadística] para averiguar si la religiosidad disminuyó. De acuerdo con los datos del IBGE de 1960, 91.21\% de la población nacional era católica; $5.30 \%$, protestante; $0.38 \%$, sin religión y $0.039 \%$ no respondió. En 2010, el censo del IBGE informa: $64.83 \%$ son católicos; $22.26 \%$, evangélicos; $0.52 \%$, luteranos; $8.039 \%$, sin religión; sin respuesta o con múltiple pertenencia religiosa, $0.337 \%$. La clasificación de las categorías ya apunta hacia una diferencia por lo que respecta a la religiosidad. El número de categorías entre 1960 y 2010 aumentó: de 8 a 22. “Tales características del censo más reciente son señales de que la definición subyacente de identidad religiosa adoptada no es la misma que se adoptó en 1960. Actualmente, la identidad religiosa parece ser vista como algo personal, fruto de la elección personal y con posibilidades de modificarse” (SEIDE, 2016: 345).

La autora también compara los datos con el censo del IBGE 2010 de Marechal Cândido Rondon: $56.21 \%$ son católicos; $38.85 \%$, evangélicos; $25.11 \%$, luteranos; $2.06 \%$, sin religión; y sin respuesta o con pertenencia religiosa múltiple, $0.22 \%$. Queda de manifiesto que los datos "sin religión" del municipio son inferiores en comparción con los datos nacionales y, por eso, alterarían la idea de que la comunidad rondonense habría tenido decremento en la religiosidad. Es por ello que la autora refuta la primera hipótesis. 
Se puede observar lo mismo con los datos generados a partir de las entrevistas aquí analizadas. Puede observarse, a continuación, la cantidad porcentual de religiones informadas en las entrevistas:

Tabla 1

Religiones mencionadas en porcentaje para cada década

\begin{tabular}{|c|c|}
\hline Década & Religiones mencionadas en porcentaje \\
\hline \multirow[t]{2}{*}{$1930 / 1940$} & Católica $-94 \%$ \\
\hline & Evangélica - 3\% Evangélica luterana - 3\% \\
\hline \multirow[t]{2}{*}{1950} & Católica $-79 \%$ \\
\hline & Evangélica $-15 \%$ Evangélica luterana $-6 \%$ \\
\hline \multirow[t]{3}{*}{1960} & Católica $-82 \%$ \\
\hline & Evangélica - $15 \%$ \\
\hline & Espiritista kardecista - 3\% \\
\hline \multirow[t]{2}{*}{1970} & Católica $-82 \%$ \\
\hline & Evangélica $-15 \%$ Evangélica luterana - 3\% \\
\hline \multirow[t]{2}{*}{1980} & Católica $-74 \%$ \\
\hline & Evangélica $-26 \%$ \\
\hline \multirow[t]{3}{*}{1990} & Católica - 59\% \\
\hline & Evangélica $-32 \%$ Evangélica luterana $-6 \%$ \\
\hline & Espiritista kardecista $-3 \%$ \\
\hline \multirow[t]{2}{*}{2000} & Católica $-68 \%$ \\
\hline & Evangélica - 23\% Evangélica luterana - 3\% \\
\hline \multirow[t]{2}{*}{2010} & Católica - 63\% \\
\hline & Evangélica $-31 \%$ Evangélica luterana $-6 \%$ \\
\hline
\end{tabular}

Fuente: elaborada por la autora (2019)

Los informantes de la investigación son, en todas las décadas, predominantemente católicos. A pesar de ser la proporción mayor, es necesario subrayar que hay oscilaciones en la categoría de los católicos dentro del corte diacrónico. El índice mayor se alcanzó en 1930/1940; a partir de 1950 se produjeron alteraciones de $3 \%$ y se mantuvo el mismo porcentaje en 1960 y 1970. En 1980, hubo un descenso de $8 \%$ y el valor más significativo se 
produjo en 1990, con otro descenso del 13\%. En 2000, hubo un aumento del $9 \%$ y en 2010, una nueva caída del 5\%.

Al paso que los católicos disminuían durante el período de análisis, los evangélicos aumentaban. En 1930/1940, apenas el 3\% representaba esa categoría; el aumento significativo ocurrió en 1950, en que el número de evangélicos creció en $12 \%$ y permaneció constante en las siguientes dos décadas. En 1980, el número se elevó hasta 26\%, esto es, un aumento del $11 \%$ con relación a la década anterior. En 1990, se produjo otro aumento del 6\%, además de aparecer otras religiones mencionadas. En 2000, hubo un decrecimiento de $9 \%$ y, finalmente, en 2010, un aumento del $8 \%$. Seide señala el mismo fenómeno, al percibir que en las últimas décadas había más categorías disponibles.

Otro factor convergente es que los datos del censo 2010 del IBGE de Marechal Cândido Rondon muestran otras categorías de religión, así como se puede ver en la tabla anterior. Además de católicos y evangélicos, en todo el lapso temporal, se pudo observar la denominación de evangélicos luteranos y espiritistas kardecistas. Se subraya que, en la investigación, a pesar de que gran parte de los informantes sean católicos, el municipio en cuestión fue colonizado principalmente por familias germánicas, donde la práctica predominante de los evangélicos era la luterana. (SAATKAMP, 1985: 207).

Sin embargo, en este corpus no hubo mención de informantes sin religión o que no dieron respuesta, lo que también puede refutar la idea de que el hecho de haber disminuido la motivación religiosa en el período de 1980 a 2000, habría disminuido la religiosidad de los habitantes del municipio.

Otro aspecto presentado por Seide, que documenta la segunda hipótesis, es que la expresión de la identidad religiosa no se expresa en la selección de los nombres religiosos dados a los hijos a partir de la década de 1980 (SEIDE, 2016: 348). El relato del alumno 
Valdiney José (nacido en la década de 1980) se emplea en esa investigación. Según él, el nombre de su padre, José (nacido entre las décadas 1950 y 1960) fue atribuido para honrar a San José, hecho éste que comprueba la tendencia de utilizar un nombre pretendidamente religioso a un hijo. En cambio, el alumno comenta que la elección del antropónimo José para él mismo le fue dado en homenaje a su propio padre y no por ser un nombre de santo, lo que connota una pertenencia a la familia y no a la religión. Así, la autora defiende

\begin{abstract}
Esa opción por no determinar la religión del hijo presupone otra relación con la religión y con la religiosidad: se trata del punto de vista según el cual la religión es una elección individual, elección que puede darse con base en un repertorio cada vez más amplio de alternativas y que puede ser también una elección provisional, dada la posibilidad, hoy en día, de que un individuo cambie de religión varias veces a lo largo de su vida (Seide, 2016: 348).
\end{abstract}

Por lo tanto, la elección de la religiosidad parte del individuo a lo largo de su vida que tiene,tambíen, libertad de cambio de religión. Si la motivación religiosa no es uno de los factores principales para atribuir un nombre a un hijo en la década de 1980 y 1990, otras maneras de nombrar a un individuo son pertinentes y pueden apuntar hacia otras cuestiones identitarias y culturales. Dick (1992) afirma: "se oculta, sin sombra de duda, en la opacidad de los registros contemporáneos, cuando los designativos [sic] son escogidos, en la mayoría de las veces, mucho más por las modas actuantes en el momento, que por cualquiera otra preocupación con su legítimo significado" (DICK, 1992, p. 181). Eso comprueba que las motivaciones para nombrar a un hijo cambian y que las modas, esto es, las tendencias antroponímicas empleadas en aquel momento histórico, pueden ser un factor de atribución.

Otro ejemplo semejante al del académico Valdiney José se observa en el corpus de esta investigación. En el conjunto de las entrevistas analizadas, hay narrativas de distintas generaciones de una misma familia. Maria Ivone, nacida en la década de 1940, en Rio Grande do Sul, vino para la ciudad de Marechal Cândido Rondon en el período de colonización a mediados de la década de los 1950. Relata la elección de su nombre: "Mire, en aquella época, en aquella región, las personas daban siempre dos nombres al hijo, y siempre nombres de 
santos, católicos ¿no? y por eso me pusieron Maria. E Ivone también es católico”. Es posible observar que tanto el primero como el segundo nombre muestran la religiosidad de la familia; queda más de relieve en el momento en que la entrevistada señala que la práctica de otorgar nombres de santos ocurría "siempre" en la región desde donde vino y que son nombres de la religión católica. Deitos (2007) explica que la iglesia católica tuvo un papel importante en la formación de las colonias de Río Grande do Sul. Tal influencia pasó a través del movimiento migratorio, cuando vinieron a la región del oeste del Paraná: “en el caso de las colonias italianas de Río Grande do Sul, la presencia del catolicismo se situaba como fuerza de nominación. También se puede relacionar esta herencia, en parte, con el proceso de colonización del oeste del Paraná” (Deitos, 2007: 1985).

El relato de Maria Ivone sobre sus dos hijas, ambas nacidas en la década de 1960, muestra cierta divergencia en la práctica nominativa. La primera, Marli Maria, fue nombrada así: "Entonces, tenía que ponerle dos nombres, decidí ponerle Maria, que es de santo ¿no?, entonces la gente iba mucho a la iglesia, y Marli fue porque tenía una cuñada que no podía hablar bien, pero sí lograba decir Marli, entonces, le puse Marli porque era un nombre fácil, que ella podría decir”. La práctica de dar nombres religiosos en la segunda generación pasa al segundo nombre. Maria Ivone nuevamente enfatiza la importancia de dar un "nombre de santo" ya que era devota y tiene la costumbre de ir a la iglesia, mientras que la elección del segundo nombre fue una cuestión estética: tenía que ser un nombre simple, de fácil pronunciación.

En cuanto al nombre de su segunda hija, Marlene Teresinha, la informante comenta: “Tenía una cuñada con el nombre de Teresinha y también es el nombre de una santa ¿no? de ahí que decidí ponerle un nombre de la familia y santo. Y Marlene fue una cuestión de gusto”. En el nombre de la segunda hija sólo se manifiesta el nombre de santo en segunda posición. 
Aunque haya una doble motivación para el nombre Teresinha, homenaje hacia la tía, el primer nombre fue nuevamente atribuido por una cuestión estética.

Mientras que la motivación religiosa atañe al primero y al segundo nombres de Maria Ivone, para sus hijas, nacidas dos décadas después, ya en la ciudad de Marechal C. Rondon, la motivación religiosa se extiende apenas para el segundo nombre. Dick (1992) asegura la tradición del uso del antropónimo Maria siempre acompañado de algún otro nombre, como, por ejemplo, Maria Rosa, Maria Antonia o incluso Maria Ivone. Las hijas, en cambio, no llevan el nombre religioso en primera posición y sí en la segunda, lo que puede ser una evidencia de cambios en la norma antroponímica.

La hija Marlene Teresinha, que reside en la ciudad, también relató la elección del nombre de sus tres hijos, João Paulo y Pedro Henrique, nacidos en la década de 1980, así como Patrícia Helena, nacida en la década de 1990. Relata así la elección del nombre de su primer hijo: "João Paulo en homenaje al abuelo materno, que es João y Paulo, en homenaje al tío paterno". En la tercera generación de la familia, los nombres otorgados por motivación religiosa no son más utilizados, aunque los nombres de João y Paulo tengan connotación religiosa. Los nombres fueron atribuidos, el primero, por un homenaje a las figuras masculinas de la familia, el abuelo y el tío. Lo mismo ocurre en el corpus de Seide (2016) con el nombre Valdiney José: nombres para enaltecer a la familia en la década de 1980.

Con relación al segundo hijo, Marlene Teresinha dijo: “el nombre Pedro Henrique fue atribuido por la numerología: se escogieron cinco nombres antes de que naciera y, de estos cinco nombres, la numeróloga actuó y encontró como mejores ésos”. El nombre del segundo hijo ya apunta hacia creencias individuales, nombres que pueden atribuir características positivas a los hijos o un buen augurio. En este sentido, Dick (1992: 191, subrayados de la autora) apunta a la necesidad desde mucho tiempo atrás de atribuir nombres: “de ahí la 
importancia de tener un "buen nombre", según las tradiciones grupales, para atraer los beneficios de un mundo superior”.

Algo semejante ocurre con el tercer hijo de Marlene Teresinha, nacida en la década posterior: "Patrícia fue en homenaje a una alumna, pues su padre era profesor, y él tenía una alumna muy aplicada, carismática y quiso rendirle homenaje a esa alumna. Y Helena fue por la numerología, entonces, de los nombres escogidos, Helena fue el que resultó ser el más apropiado". Las dos últimas narrativas ponen en evidencia la idea de que otras motivaciones pueden ser interesantes para nombrar a un hijo, ya sea el rendir homenaje a la familia, a un conocido, o bien, creencias individuales de los padres.

Es claro, entonces que la práctica de nominación cambió en tres generaciones: en 1930/40, con la narrativa de Maria Ivone sobre su propio nombre, su narrativa en relación con los nombres de sus hijas, nacidas en 1960, y la narrativa de Marlene Teresinha, hija de Maria Ivone, que relata la selección de los nombres de sus hijos, nacidos en 1980 y 1990.

Para tener una panorámica más amplia, al tiempo que nombres cuya motivación religiosa disminuyó, otras motivaciones son mencionadas. Tales motivaciones, en gran parte debidas a modas, también pueden poner de relieve diferencias en las elecciones antroponímicas. Aunque no sea el objeto de este estudio, cabe mencionar aquí las motivaciones más empleadas en el período en que la religiosa no obtuvo porcentajes significativos. En los antropónimos femeninos, como ya se mencionó, en la década de 1990. no hubo ningún caso ni en n1 ni como n2, en cambio, los nombres de moda, influidos por los medios de comunicación masiva y la heterogeneidad cultural fueron ampliamente mencionados (66\% en n1 y en n2). Al paso que, en los antropónimos masculinos, el intervalo sin motivación religiosa se produjo en el n2, en tres décadas consecutivas, 1970, 1980 y 1990. En la primera se destacaron los nombres escogidos en homenaje a la familia y los nombres 
de moda (35\% y $23 \%$ respectivamente), en la segunda, el uso de la motivación estética y los medios de comunicación masiva $(22 \%$ y $26 \%)$ y, en la última, la misma motivación de la década anterior $(41 \%$ y $6 \%)$.

\section{Consideraciones finales}

En esta investigación se analizó si nombres cuya motivación es religiosa son el reflejo de una sociedad más religiosa en el municipio de Marechal Cândido Rondon. Los nombres yuxtapuestos analizados en el corte diacrónico mostraron que se prefiere atribuir nombres religiosos en la década de 1930/40, mientras que, en la década de 1990, se presentan otras motivaciones.

A pesar de que se mencione la motivación religiosa en menores ocurrencias en algunas décadas (1980, 1990), no se confirma el hecho de que la religiosidad del municipio se haya reducido. Además de eso, es claramente observable que la identidad religiosa puede no ser evidente en los antropónimos, y sí en otras elecciones realizadas por el individuo. La entrevistas realizadas en la familia de Maria Ivone comprueban que la identidad religiosa expresada antroponímicamente no pasó a las dos generaciones posteriores.

Más aun, desde el punto de vista socioantroponímico, estos datos revelan que la elección de nombres religiosos, por ejemplo, ponen de relieve otra marca identitaria; la cultura de los inmigrantes traída de sus países de origen (DEIDE, 2020b). En el contacto con un nuevo contexto social, nuevas motivaciones se emplean para dar nombres, lo que puede apuntar hacia una necesidad de los padres de hacer que los hijos sean identificados como pertenecientes a la nueva comunidad (SEIDE, 2020b: 102). 
Aunque la investigación haya analizado cuestiones como la religiosidad y la identidad religiosa dentro del campo de estudios de la socionomástica, se necesitan todavía más estudios con el fin de poder conocer mejor aspectos sociales y culturales de la ciudad de Marechal Cândido Rondon.

Recibido el 31/08/2020

Aceptado el 29/09/2020

Publicado el 17/12/2020

\section{Referencias}

Deitos, N. J. (2007). A igreja católica no Oeste do Paraná e sua atuação no processo de colonização. Migrações e a construção do Oeste do Paraná: século XXI em perspectiva. Cascavel: Coluna do Saber. 183-189.

Dick, M. V. de P. do A. (1992) Toponímia e Antroponímia no Brasil. São Paulo: Coletânea de Estudos. FFLCH.

Guérios, R. F. M. (1981). Dicionário etimológico de nomes e sobrenomes. São Paulo: Ave Maria.

Hall, S. (1992). A Identidade Cultural na pós-modernidade. Rio de Janeiro: DP\&.

Jiménez Segura, S. (2016) Los modelos de atribuición del nombre de pila tradicional y a partir de la moda en el municipio de Tlalnepantla de Baz, estado de México. Estudio sincrónico y diacrónico de tres calas: 1930, 1960 y 1990. (Dissertação de Mestrado), Escuela Nacional de Antropología e Historia.

López Franco. Y. G. (2014). Los nombres de pila en la década de 1980 en Montpellier, Francia, y en Tlalnepantla de Baz, México, bajo un enfoque socioantroponímico. In. Isquerdo, A.N.; Dal Corno, G.O.M, As ciências do léxico. Lexicologia, lexicografia, terminologia, Campo Grande: Ed. UFMS.

Pensin, T.G. (2020). Uma revisão ao perfil nomeador dos habitantes de Toledo, Paraná:tradicional ou inovador? Onomástica desde América Latina, n.1, v.1. 199-221.

Saatkamp, V. (1985). Desafios Lutas e Conquistas: História de Marechal Cândido Rondon. Cascavel: Assoeste.

Seide, M. S. (2016). A identidade religiosa na antroponímia de Marechal Cândido 
Onomástica Desde América Latina, n.3, v.2, janeiro - junho, 2021, p. 177 - 198 ISSN 2675-2719 https://doi.org/10.48075/odal.v2i3.26603

Rondon.

Revista Relin, v. 24, n. 1. 167-186.

Seide, M. S. (2018). Moda e tradição na antroponímia. A.N.; Dal Corno, G.O.M, As ciências do léxico. Lexicologia, lexicografia, terminologia Campo Grande: Ed. UFMS. $161-178$.

Seide, M. S. (2020a). A Antroponomástica Comparada. Onomástica desde América Latina, n.2, v.1. 83-102

Seide, M. S. (2020b). Antroponimia, diáspora y migración: los descendientes de lituanos en Brasil. Onomástica desde América Latina, n.1, v.1. 100-121

Van Langendonck, W. (2007). Theory and Typology of Proper Names. Berlin/New York: Mouton de Gruyter. 